\title{
Fields of efficiency of breeding methods for per se value or combining ability in plant breeding
}

\author{
A Gallais 1,2 \\ 1 INRA-UPS, Station de Génétique Végétale, ferme du Moulon, F91190 Gif-sur-Yvette; \\ 2 INA P-G, 16, rue Claude-Bernard, F75231 Paris Cedex 05, France
}

(Received 7 November 1991; accepted 30 April 1993)

\begin{abstract}
Summary - Starting from classical expressions of genetic advance for per se value or general combining ability, the fields of efficiency of 6 breeding methods (mass selection, half-sib family selection, full-sib family selection, half-sib progeny test, full-sib progeny test and $S_{1}$ selection) were determined with the following assumptions: no epistasis, absence of genotype $x$ environment interaction, same number of plants at the level of the test whatever the selection methods and same effective size in one cycle. Genetic advance was considered at 3 levels: per cycle, per generation and per unit of time with one off-season generation for intercrossing. To simplify the comparisons among methods involving families, a sufficiently large number of plants per family was considered, which permitted an examination of relationships between the heritabilities of the various testing systems. For the 5 breeding methods involving family or progeny testing, 2 types of 'fields' were determined, those that were a function of heritability and the correlation $\rho_{s_{1}}$ between additive effect for $S_{1}$ value and additive effect for per se value (for a given part of the additive variance $\left(a^{2}\right)$ and a given ratio $r_{S_{1}}^{2}$ of additive variance in $S_{1}$ to classical additive variance), and those that were a function of heritability and of the part of additive variance (for given values of $\rho_{\mathrm{S}_{1}}^{2}$ and ${f_{\mathrm{S}}}_{\mathrm{S}_{1}}$ ). On a per cycle basis, among methods with progeny and family testing, $S_{1}$ selection is the best in a large range of situations from medium to low heritabilities. Per unit of time, without and more clearly with one off-season generation for intercrossing, the field of efficiency of $S_{1}$ selection restricted and full-sib family recurrent selection is the most efficient in a large range of realistic situations. From comparisons involving mass selection and family selection without an off-season generation, it appears that mass selection is the best method in several situations, not necessarily at high heritability. However, the advantage of mass selection at medium to low heritability disappear with the use of one off-season generation for intercrossing in family selection.
\end{abstract}

breeding method / mass selection / progeny selection / family selection / genetic advance

Résumé - Domaine d'efficacité des méthodes de sélection pour la valeur propre ou l'aptitude à la combinaison en amélioration des plantes. À partir des expressions classiques du progrès génétique sur la valeur propre ou sur la valeur en combinaison, les domaines d'efficacité de six méthodes de sélection (sélection massale, sélection familiale demi-frères, sélection familiale pleins-frères, sélection sur descendances pleins-frères et sélection sur descendances $S_{1}$ ) sont déterminés avec les hypothèses suivantes : absence d'épistasie, absence d'interaction génotype $x$ milieu, même nombre de plantes au niveau du test, quelle que soit la méthode, et même taille génétique effective en un cycle. Le progrès génétique est considéré à trois niveaux : par cycle, par génération et par unité de temps avec une génération hors-saison pour l'intercroisement. Pour simplifier les comparaisons impliquant des méthodes avec test de familles, un nombre suffisamment grand de plantes par parcelle est considéré ; cela permet de relier entre elles les héritabilités des différents systèmes de test. Pour les cinq méthodes impliquant des familles, deux types de domaines sont déterminés, ceux qui sont une fonction de l'héritabilité et de la corrélation $\left(\rho_{s}\right)$ entre les effets additifs pour la valeur propre et pour la valeur en $S_{1}$ (pour une proportion donnée de la variance d'additivité $\left(a^{2}\right)$ et pour un rapport donné $r_{S_{1}}^{2}$ de la variance d'additivité en $S_{1}$ à la variance additivité classique), et ceux qui sont une fonction de l'héritabilité et de la part de la variance d'additivité (pour des valeurs fixées de $\rho_{S_{1}}^{2}$ ). Parmi les méthodes avec test de descendances ou de familles, la sélection sur descendances $S_{1}$ est la meilleure pour le progrès génétique par cycle et cela pour une large gamme de situations allant d'héritabilités moyennes à faibles. Par unité de temps, sans et plus nettement avec, une génération hors saison pour l'intercroisement, le domaine d'efficacité de la sélection sur descendances $S_{1}$ est plus étroit, et la sélection familiale pleins-frères est la plus efficace dans une large gamme de situations réalistes. À partir des comparaisons impliquant la sélection massale et la sélection familiale, 
sans génération hors-saison, il apparaît que la sélection massale peut être la meilleure méthode dans plusieurs situations et non pas nécessairement à forte héritabilité. Cependant l'avantage de la sélection massale pour des héritabilités moyennes à faibles disparaît avec l'utilisation d'une génération hors saison pour l'intercroisement de la sélection familiale.

méthode de sélection / sélection massale / sélection sur descendances / sélection familiale / progrès génétique

\section{INTRODUCTION}

In plant breeding theory, formulae for genetic advance in per se value or in combining ability for different selection schemes that do not use inbreeding at the testing level are classical (Sprague, 1966; Hallauer and Miranda, 1981). Since the development of the theory of covariances between inbred relatives (Gillois, 1964 ; Harris, 1964) it is also possible to express genetic advance with $S_{1}$-recurrent selection in terms of the genetic effects that are involved. Classically the formulae are given per cycle and sometimes per generation. For the plant breeder to choose a given method, it would be interesting to know the situations wherein a given scheme is better than another per unit of time or per unit of means. However, from the formulae of genetic advance per cycle except in some situations, it is difficult to directly compare the various methods for their relative efficiency. Some simulation studies (Choo and Kannenberg, 1979; Wright, 1981) have attempted to compare various methods. The main advantage of simulation studies is the possibility to predict long-term advance. However, it is not always clear from the results what the effects of the parameters of genetic advance are. Furthermore, experimental comparisons of breeding methods are difficult or even impossible to develop with sufficient accuracy to obtain a sufficiently general meaning.

Considering the response to selection in only the first cycles ( 1 to 3 , according to the length of the cycle; for example, for an annual plant, $1 \mathrm{cy}$ cle of a method with progeny-testing and 2-3 cycles of mass selection) numerical application from the formulae of expected genetic advance can lead to clearer conclusions for the plant breeder. Indeed, with given values of the parameters determining genetic advance (which may be known or estimated by the plant breeder) it is possible to compare the genetic advances of the methods considered at different levels:

- per cycle: but this is not meaningful when the length of the cycle differs from one method to another ;
- per generation: this is more meaningful than the previous level of comparison but does not consider the possibility for some selection schemes to accept off-season generations;

- per unit of time: with or without 1 off-season generation for intercrossing ;

- per unit of cost: in a given time, this could be one of the more interesting levels of comparison. However, such an approach can only be specific to a firm, because the cost parameters depend on the organization and the size of the enterprise. Furthermore, it is also necessary to consider the benefit provided by a greater genetic advance which is even more expensive but leads to a quicker development of a good variety which will be rapidly adopted on the market. With the consideration of only the cost of genetic advance in population improvement, the most efficient method could be that leading to the lowest genetic advance, as it will be very cheap. Thus to answer correctly, it is necessary to develop a general economy of plant breeding.

A simplified consideration of the cost could be to impose constraints to methods in order to obtain the same cost in a given time. However, such an attitude does not consider the possibility for some methods to accept more investment: this is the case of methods with a short cycle. For example, for an annual plant without an offseason generation, in 3 years it is possible to develop 3 cycles of mass selection and only 1 cycle of a scheme with progeny-testing; then for a given capacity of test in a given year, it is possible to study more plants with mass selection than with progeny testing.

Investment in the testing capacity can affect the selection intensity. Indeed, this parameter of genetic advance is determined by the number of units tested which is related to the testing capacity, and by the number of units selected which cannot be too low in order to obtain the best use of genetic variance. Selection intensity has to be adapted to the selection scheme by consideration of the decrease in genetic variance. The number of intercrossed units determines the effective size of the population which can be an in- 
dicator of the variance. Then it could be interesting to compare the methods in such a way that the effective size will be the same in a given time. Another approach could be to consider formulae for the decrease in genetic variance, and to compare the methods for the same decrease of genetic variance in a given time. However, the formulae have only been developed for the case of additivity and are valid only over a short-term period (Bulmer, 1980; Verrier et al, 1990).

From the breeder's point of view, such comparisons on the basis of the same reduction of effective size or variance per unit of time are not meaningful. Indeed, genetic advance can only occur at the expense of genetic variability. As the greater genetic advance per unit of time will generally be associated with the greater decrease in variance per unit of time, it then appears better to consider the modalities of the schemes which lead to the best use of possible resources and genetic variance in a given time. From this point of view, it seems justified to compare the methods on the basis of the same effective number of intercrossed plants at the first cycle. This is all the more justified as short-term response is considered. In practice, as recurrent selection is not a closed system, the plant breeder is not really concerned by long-term response on a constant genetic base. It must also be noted, however, that the effective number of plants intercrossed has to be related to heritability: to limit the loss of favourable genes by genetic drift it must be relatively higher at low than at high $h^{2}$.

Comparison between selection schemes is difficult because of the large number of parameters and the different possible attitudes or questions or levels of evaluation of relative efficiency. Experimentally it is better to estimate the biometrical parameters of the response to selection and to predict the response in one cycle according to the value of all the parameters (selection intensity, testing system, etc). This is the approach adopted in this paper via numerical application from the formulae of genetic advance in one cycle.

Starting with classical formulae, the aim of this study is to determine the domains of efficiency of several selection schemes, per cycle, per generation or per unit of time with the use of 1 offseason generation for intercrossing. Six intrapopulation schemes are considered: mass selection (M), half-sib family selection (HSF-RS), full-sib family selection (FSF-RS), half-sib progeny selection (HSP-RS), full-sib progeny selection
(FSP-RS) and $S_{1}$ selection ( $\left.S_{1}-R S\right)$. Half-sib and full-sib family and progeny selections use the same testing system but the schemes differ by the units that are intercrossed: families in family selection and parental plants in progeny selection.

\section{The assumptions}

To simplify the approach it is assumed that there is no genotype $x$ environment interaction or that the environment can be considered approximately constant or repeatable (as in the case of plants grown under controlled environments, as in glasshouses). With the approach considered it is possible to introduce the effect of genotype $x$ environment interaction. This will increase the number of parameters; in a preliminary step it is interesting to discuss the relative efficiency in a given environment.

It is also assumed that there is no epistasis (or that the contribution of epistasis to the genetic advance can be neglected). This decreases the number of parameters. Furthermore, in the absence of epistasis, the expected genetic advance in per se value and general combining ability is the same (Gallais, 1989). Another consequence is the equality of genetic advance per cycle of FSF-RS and FSP-RS (if there is the same selection intensity). The presence of epistasis will not qualitatively change the results.

The same number of plants is considered at the level of the testing system. Then in a given time, this leads one to allocate more means to schemes with a short cycle; this is justified because when investment in space is limited, some schemes allow more investment in time. Note that, according to the species, the cost of one plant can vary from one scheme to another (for example, in some situations, the cost of one plant in mass selection can be greater than the cost of one plant in progeny testing due to a plot evaluation; then for the same investment, more plants could be established with progeny testing).

A consequence of the assumption on the testing capacity is that the selection intensities are determined by the structure of the testing design and by the number of selected units. With the constraint of the same effective size, this leads to 4 types of selection intensity: $i_{M}$ for mass selection, $i_{F S}$ for FSF-RS and FSP-RS, $i_{H S}$ for HSF-RS, and $i_{H P}$ for HSP-RS and $S_{1}-R S$. For a 
given number of plants in the testing design, relationships between $i_{M}, i_{H F}, i_{H P}$ and $i_{F S}$ can be established from the corresponding rates of selection $p_{M}, p_{H S F}, p_{H S P}$ and $p_{F S F}$. Taking HSPRS as a reference, it results that $p_{F S F}=p_{H S P} / 2$, $p_{H S F}=p_{H S P} / 4$ and $p_{M}=p_{H S P} / N$ where $N$ is the number of plants per family. To illustrate the relationship between $i_{H P}$ and $i_{H M}$ consider the situation with a capacity of 10000 plants and selection of 50 plants in mass selection, $i_{M}$ will be 2.89. Now with a family structure at the same plant density and 50 plants per family, 200 families can be studied ; if 50 plants are selected, $i_{H P}$ will be 1.27 .

For the comparison of mass selection to the other methods, it must be considered that in practice, for the schemes with family or progeny testing, there are 2 levels of selection: first, selection at the level of the mother plants for their phenotypic value and second a selection at the level of family. Only the second level is considered in table I. If the first level can be ignored for the comparison among schemes with family or progeny testing because its contribution in $1 \mathrm{cy}$ cle is the same whatever the scheme, it cannot be ignored for comparison with mass selection. The difficulty is then to consider the effect of the phenotypic selection on the genotypic variance, a problem which is not analytically well resolved. However, when all the variance is additive, it can be shown (Bulmer, 1980) that the ratio of the new variance to the original variance will be [1 $\left.i_{P}\left(i_{P}-x_{P}\right) h^{2}\right]$ where $x_{P}$ is the abscissa of the truncation point of the standardized distribution, and $h^{2}$ the heritability.

Table I. Expressions of genetic advance for various breeding methods.

Breeding method

Genetic advance per cycle

(1)

(2)
Cycle length Genetic advance per unit of time

(3) (4)

Without off-season With one off-season

(6)

1. Mass selection $\theta=1$

$$
\theta=2
$$

2. HS family selection $(\theta=2)$ (HSF-RS)

3. FS family selection (FSF-RS)

4. HS progeny selection (HSP-RS)

5. FS progeny selection (FSP-RS)

$$
i_{M} \frac{1 / 2 \sigma_{A}^{2}}{\sqrt{ } \operatorname{var} P} \quad i_{M} 1 / 2 h_{M} \sigma_{A} \quad 1 \quad 1 \quad i_{M} 1 / 2 h_{M} \sigma_{A} \quad i_{M} 1 / 2 h_{M} \sigma_{A}
$$

$i_{M} \frac{\sigma_{A}^{2}}{\operatorname{var} P} \quad i_{M} h_{M} \sigma_{A} \quad 2^{\star} \quad 1 \quad i_{M} 1 / 2 h_{M} \sigma_{A} \quad i_{M} h_{M} \sigma_{A}$
$i_{H F} \frac{1 / 4 \sigma_{A}^{2}}{\sqrt{\operatorname{var} P_{H S}^{-}}} \quad i_{H F} 1 / 2 h_{H S} \sigma_{A} \quad 2 \quad 1 \quad i_{H F} 1 / 4 h_{H S} \sigma_{A} \quad i_{H F} 1 / 2 h_{H S} \sigma_{A}$ $i_{F S} \frac{1 / 2 \sigma_{A}^{2}}{\sqrt{\operatorname{var} P_{F S}}} \quad i_{F S} \sqrt{2} / 2 h_{F S} \sigma_{A} \quad 2 \quad 1 \quad i_{F S} \sqrt{2} / 4 h_{F S} \sigma_{A} \quad i_{F S} \sqrt{2} 2 / 2 h_{F S} \sigma_{A}$ $i_{H P} \frac{1 / 2 \sigma_{A}^{2}}{\sqrt{v a r} P_{H S}} \quad i_{H P} h_{H S} \sigma_{A} \quad 3 \quad 2 \quad i_{H P} 1 / 3 h_{H S} \sigma_{A} \quad i_{H P} 1 / 2 h_{H S} \sigma_{A}$ $i_{F S} \frac{1 / 2 \sigma_{A}^{2}}{\sqrt{\operatorname{var} P_{F S}}} \quad i_{F S} \sqrt{2} / 2 h_{F S} \sigma_{A} \quad 3 \quad 2 \quad i_{F S} \sqrt{2} / 6 h_{F S} \sigma_{A} \quad i_{F S} \sqrt{2} / 4 h_{F S} \sigma_{A}$ 6. Progeny (or family) selection $i_{H P} \frac{\sigma_{A \cdot A_{s_{1}}}}{\sqrt{ } \operatorname{var} P_{S_{1}}} \quad i_{H P} \rho_{S_{1}} h_{s_{1}} \sigma_{A} \quad 3 \quad 2 \quad i_{H P} 1 / 3 r_{S_{1}} h_{S_{1}} \sigma_{A} \quad i_{H P} 1 / 2 \rho_{S_{1}} h_{s_{1}} \sigma_{A}$
$\left(S_{1}-\mathrm{RS}\right)$

* If intercrossing is not possible in the generation of selection. (3) Without off-season generation; (4) with off-season generation for intercrossing. 


\section{THE EXPRESSIONS OF GENETIC ADVANCE AND HERITABILITIES}

Table I gives the expressions of genetic advance for per se value on a per cycle basis and per unit of time. Note that in the absence of epistasis, the genetic advance in per se value is equal to genetic advance in general combining ability. Two types of expressions are given. In column (1) are classical expressions of genetic advance per cycle (Sprague, 1966; Hallauer and Miranda, 1981 ; Gallais, 1989). For $S_{1}-R S, \sigma_{A . A_{s 1}}$ is the covariance between additive effect for per se value and additive effect for $S_{1}$ value (Gallais, 1988, $1989)$; it can be also written:

$$
\sigma_{A . A_{s_{1}}}=\sigma_{A}^{2}+\sigma_{A D},
$$

where $\sigma_{A D}$ is the covariance additivitydominance due to inbreeding (Gillois, 1964 ; Harris, 1964). Each method is characterized by the phenotypic variance of the tested units. In column (2) the expressions are given in terms of the narrow sense heritability associated with the testing system. For a method $x$ the general formula is of the type (Gallais, 1989):

$$
\Delta G_{x}=i_{x} \theta_{x} \rho_{x} h_{x} \sigma_{M x}
$$

$-i_{x}$ is the selection intensity ;

$-\theta_{x}$ is the degree of control of the selection on the 2 sexes;

$-\rho_{x}$ is the correlation between the additive effect for the testing system of the parents and the additive effect for per se value: $\rho_{x}=1$ for all schemes except for $S_{1}$-RS ;

- $h_{x}^{2}$ is the narrow sense heritability: $h_{x}^{2}=\sigma_{A_{x}}^{2}$ / $\sigma_{P_{x}}^{2}$ where $\sigma_{A_{x}}^{2}$ is the additive variance for the testing system. For all methods, except $S_{1}-R S$, $h_{x}^{2}$ is also the part of the phenotypic variance among tested units due to additivity;

$-\sigma_{M x}^{2}$ is the genetic variance among progenies from intercrossed units:

$\sigma_{M x}^{2}=1 / 4 \quad \sigma_{A}^{2}$ when the units tested and intercrossed are plants,

$\sigma_{M x}^{2}=1 / 16 \sigma_{A}^{2}$ for the half-sib family selection,

$\sigma_{M x}^{2}=1 / 8 \sigma_{A}^{2}$ for full-sib testing.

For methods 1 to 5 , it is then possible to write:

$$
\Delta G_{x}=i_{X} \theta_{x} c_{X} h_{x} \sigma_{A}
$$

where $c_{\chi}$ is the coefficient of $\sigma_{A}$ in $\sigma_{M}$.
For method $6\left(\mathrm{~S}_{1}-\mathrm{RS}\right)$ :

$$
\Delta G_{S_{1}}=\mathrm{i}_{S_{1}} \theta_{S_{1}} \rho_{S_{1}} h_{S_{1}} \sigma_{A} / 2
$$

Expressions [2] and [3] are very useful for the comparison of the methods because the ratio of genetic advance for any pair of methods eliminates the additive variance. Then, only the relative parameters $\left(h_{x}, \rho_{x}\right)$ or parameters that are known $\left(c_{x}\right)$ or that can be known (selection intensities) remain.

The genetic advance per unit of time (columns 5 and 6 , table I) are derived by dividing expressions [2] and [3] by the length of the cycle $t_{x}$. Column 3 , table I, gives the length of the cycle considered for each breeding scheme. When one off-season generation is considered, it is assumed that only intercrossing can be developed in such conditions.

Then considering expressions of genetic advance in table $I$ in columns 2,5 and 6 , and as $\sigma_{A}$ is a common parameter, to develop a priori comparisons of the breeding methods it is necessary to know their associated selection intensities and heritabilities and the coefficient of correlation $\rho_{s_{1}}$ between additive effects for per se value and additive effect for $S_{1}$ value if $S_{1}-R S$ is included in the comparison.

\section{Expressions of heritabilities}

Four heritabilities are to be considered: $h_{\mathcal{M}}^{2}$, $h_{H S}^{2}, h_{F S}^{2}$ and $h_{s_{1}}^{2}$. In terms of genetic parameters :

$$
h_{M}^{2}=\frac{\sigma_{A}^{2}}{\sigma_{G}^{2}+\sigma_{e}^{2}}
$$

$$
h_{H S}^{2}=\frac{1 / 4 \sigma_{A}^{2}}{\left.1 / 4 \sigma_{A}^{2}+1 / b\left\{\sigma_{p}^{2}+\left(\sigma_{W H S}^{2}+\sigma_{\mathrm{eW}}^{2}\right) / n\right)\right\}},
$$$$
\sigma_{W H S}^{2}=\sigma_{G}^{2}-1 / 4 \sigma_{A}^{2}
$$

$$
\begin{aligned}
& h_{F S}^{2}=\frac{1 / 2 \sigma_{A}^{2}}{\left.1 / 2 \sigma_{A}^{2}+1 / 4 \sigma_{D}^{2}+1 / b\left\{\sigma_{p}^{2}+\left(\sigma_{W F S}^{2}+\sigma_{e W}^{2}\right) / n\right)\right\}}, \\
& \sigma_{W F S}^{2}=1 / 2 \sigma_{A}^{2}+3 / 4 \sigma_{D}^{2}
\end{aligned}
$$




$$
\begin{aligned}
& h_{s 1}^{2}=\frac{\sigma_{A_{s 1}}^{2}}{\sigma_{A_{s 1}}^{2}+1 / 4 \sigma_{D}^{2}+1 / b\left\{\left(\sigma_{p}^{2}+\left(\sigma_{W S_{1}}^{2}+\sigma_{e W}^{2}\right) / n\right\}\right.}, \\
& \sigma_{W S_{1}}^{2}=\sigma_{T S_{1}}^{2}-\sigma_{A_{S 1}}^{2}
\end{aligned}
$$

where $b$ is the number of replications, $n$ is the number of plants per plot, $\sigma_{p}^{2}$ is the between plot variance due to environment and $\sigma_{\mathrm{e} W}^{2}$ is the within plot variance due to environment $\left(\sigma_{p}^{2}+\sigma_{e W}^{2}=\right.$ $\sigma_{e}^{2}$ the environmental variance involved with mass selection). $\sigma_{A_{s 1}}^{2}$ is the variance of additive effect for $S_{1}$ value (Gallais, 1988). $\sigma_{T S_{1}}^{2}$ is the total genotypic variance among $S_{1}$ plants.

Expressions of heritabilities can be transformed in order to have relative parameters in place of variance components. The following relative parameters are introduced:

$$
\begin{aligned}
& h^{2}=\frac{\sigma_{G}^{2}}{\sigma_{G}^{2}+\sigma_{e}^{2}} \text { so } \frac{\sigma_{e}^{2}}{\sigma_{G}^{2}}=\frac{\sigma_{p}^{2}+\sigma_{e W}^{2}}{\sigma_{G}^{2}}=\frac{1-h^{2}}{h^{2}} \\
& a^{2}=\frac{\sigma_{A}^{2}}{\sigma_{G}^{2}} \text { so } h_{M}^{2}=a^{2} h^{2} ; \frac{\sigma_{D}^{2}}{\sigma_{A}^{2}}=\frac{1-a^{2}}{a^{2}} \\
& r=\frac{\sigma_{p}^{2}}{\sigma_{p}^{2}+\sigma_{e W}^{2}} \\
& \text { so } \frac{\sigma_{p}^{2}}{\sigma_{A}^{2}}=r^{2} \frac{1-h^{2}}{a^{2} h^{2}}=s_{p}^{2} \frac{\sigma_{e W}^{2}}{\sigma_{A}^{2}}=\left(1-r^{2}\right) \frac{1-h^{2}}{a^{2} h^{2}}=\sigma_{e W}^{2} \\
& t=\frac{\sigma_{A s_{1}}^{2}}{\sigma_{G T S_{1}}^{2}}, \quad r_{s_{1}}^{2} \frac{\sigma_{A s_{1}}^{2}}{\sigma_{A}^{2}}
\end{aligned}
$$

Then it results:

$$
\begin{aligned}
& h_{H S}^{2}=\frac{1 / 4}{1 / 4+\left\{s_{p}^{2}+\left[\left(1 / a^{2}-1 / 4\right)+s_{e W}^{2}\right] / n\right\} b}[5] \\
& h_{F S}^{2}=\frac{1 / 2}{1 / 4+1 /\left(4 a^{2}\right)+\left\{s_{p}^{2}+\left[3 /\left(4 a^{2}\right)-1 / 4+s_{e W}^{2}\right] / n\right\} / b}[6] \\
& h_{s 1}^{2}=\frac{r_{s 1}^{2}}{r_{s_{1}}^{2}+1 / 4\left(1-a^{2}\right) / a^{2}+\left\{s_{p}^{2}+\left[(1-t) / t r_{s 1}^{2}+s_{e w}^{2}\right] / n\right\} / b}
\end{aligned}
$$

$h_{F S}^{2}$ and $h_{\mathcal{S}_{1}}^{2}$ can be expressed in terms of $h_{H S}^{2}$ :

$$
h_{F S}^{2}=\frac{2 a^{2} h_{H S}^{2}}{a^{2}+(1-1 / b n) h_{H S}^{2}}
$$

$$
\begin{aligned}
& \text { and } h_{s_{1}}^{2}=\frac{4 r_{s_{1}}^{2} a^{2} h_{H S}^{2}}{\left\{\left(4 r_{s_{1}}^{2}-2\right)+\left[4 r_{s_{1}}^{2}(1-t) / t+1\right] / b n\right\}} \\
& a^{2} h_{H S}^{2}+a^{2}+(1-4 / b n) h_{H S}^{2}
\end{aligned}
$$

Note that when $b n=N$, the total number of plants per family, is sufficiently great, these expressions are simplified:

$$
\begin{aligned}
& h_{F S}^{2} \approx \frac{2 a^{2} h_{H S}^{2}}{a^{2}+h_{H S}^{2}} \\
& h_{s_{1}}^{2} \approx \frac{4 r_{s_{1}}^{2} a^{2} h_{H S}^{2}}{\left(4 r_{s_{1}}^{2}-2\right) a^{2} h_{H S}^{2}+a^{2}+h_{H S}^{2}}
\end{aligned}
$$

To evaluate the acceptable threshold of $N$, compute the ratio of the exact value with $1 / N \# 0$ to the value for $1 / N=0$. Consider first the case of $h_{F S}^{2}$. The ratio will be:

$$
\frac{a^{2}+h_{H S}^{2}}{a^{2}+(1-1 / M) h_{H S}^{2}}
$$

which must be desired to be less than threshold $B$ (for example $B=1.10$ means that there will be an accepted error of $10 \%$ ), then it is necessary to have:

$$
N>B /(B-1) h_{H S}^{2}\left(a^{2}+h_{H S}^{2}\right)
$$

$N$ must be greater for high heritability and low part of additive variance. Thus with $B=1.10, h_{H S}^{2}$ 0.50 and $a^{2}=0.30$, bn must be only greater than 10 , which is relatively low. With $N=50$ the greatest error for $h_{H S}^{2}=0.9$ and $a^{2}=0.1$ will be only $2 \%$. Now for $h_{S 1}^{2}$ the ratio can be greater or less than 1, so for a given $B$ it is necessary to have:

$$
N>[B / A b s(B-1)]\left[\operatorname{Abs}\left(T a^{2}-4\right)\right] h_{H S}^{2}\left(R a_{H S}^{2}\right)+a^{2}+h_{H S}^{2}
$$

with $R=4 r_{s 1}^{2}-2, T=4 r_{s 1}^{2}(1-t) / t+1 .(\operatorname{Abs}(x)$ means absolute value of $x$ ). If $R a^{2}-4=0$, the 2 values will be identical. With $t=0.66, r_{S 1}^{2}=0.81$, $T=2.62, R=1.24$, and with $B=0.90\left(T^{2}-4<\right.$ $0), h_{H S}^{2}=0.50$ and $a^{2}=0.30$, again it is found 
that if $N>10$ the bias will be lower than the threshold value. With $h_{H S}^{2}=0.9$ and $a^{2}=0.10$ (a set of values that increases the bias) it is required that $N>25$. With this set of values and with $N=50$, the error due to approximation will be $5.5 \%$. Then with a realistic range of variation of $h_{H S}^{2}$ and $a^{2}$, with $N=50$, the error will be $<5 \%$. In the following it will be assumed that $N>50$.

This simplification reduces to 5 the number of parameters necessary to study the relative efficiency of the breeding methods considered: the intensity of selection, heritability $h_{H S}^{2}, a^{2}, r_{s_{1}}^{2}$ and $\rho_{s_{1}}$. The parameters $r_{s_{1}}^{2}$ and $\rho_{s_{1}}$ will be determined from $r_{L}^{2}$ the ratio of additive variance for line value $\left(\sigma_{A L}^{2}\right)$ to the classical additive variance, and $\rho_{\mathrm{L}}$ the correlation coefficient between additive effect for per se value and additive effect for line value. From the expressions of $\sigma^{2}{ }_{A s}$, $\sigma_{A_{S 1}}^{2}, \sigma_{A}^{2}$ and of $\sigma_{A_{L}}^{2}$ given by Gallais (1988), it can be shown that in the absence of epistasis:

$r_{s_{1}}^{2}=\left(2 \rho_{L} r_{L}+r_{L}^{2}+1\right) / 4$

$\rho_{S_{1}}=\left(1+\rho_{L} r_{L}\right) / \sqrt{1+2 \rho_{L} r_{L}+r_{L}^{2}}$

The expression of the correlation $\rho_{s_{1}}$ shows that if $\rho_{L}$ is assumed positive or zero, a minimum value of $\rho_{s_{1}}$ will be $1 / \sqrt{\left(1+r_{L}^{2}\right)}$ or 0.707 for $r_{L}^{2}=1$. Indeed, examination of the results for biallelism (Gallais, 1990) shows that with partial to complete dominance, $\rho_{s_{1}}$ is expected to be very high $(\approx 1)$. With the same gene effects and gene frequencies at all loci involved, it is equal to 1 . In the absence of epistasis, low values for $\rho_{s_{1}}$ can be observed only with strong overdominance and non-uniform distribution. The ratio $r_{s_{1}}^{2}$ can be greater or less than 1. In the case of biallelism, for one locus, $r_{L}^{2}$ is greater or less than 1 when the frequency of the favorable gene is greater (case of selected populations) or less than 0.5 (case of unselected populations). It is expected that there will be a greater variation in $r_{s_{1}}^{2}$ than in $\rho_{L}$.

\section{COMPARISONS AMONG METHODS WITH PROGENY OR FAMILY TESTING}

\section{Comparison on a per cycle basis}

Fields $f\left(h_{H S}^{2}, \rho_{L}\right)$ in figure $1 \mathrm{~b}$ are established for $a^{2}=0.50, r_{L}^{2}=1.50$ and $p_{H S} 0.20 . S_{1}$-RS is the best method for medium to low heritability $\left(h_{H S}^{2}\right.$ and for medium to high value of $\rho_{L}$. For low herit- abilities $\left(h_{H S}^{2}<0.30\right)$ and low value of $\rho_{L}$, FSF$R S$ is the best method. The remaining field is the field of efficiency of HSP-RS for medium to high heritability and medium to low correlation $\rho_{L}$. A lower value of $a^{2}$ decreases the field of efficiency of $\mathrm{S}_{1}$-RS and increases that of HSP-RS.

Fields $f\left(h_{H S}^{2}, a^{2}\right)$ in figure 1a are established for $\rho_{L}=0.70$ and for $r_{L}^{2}=1.50$. $S_{1}$-RS appears to be the best method for medium to high additivity and for medium to low heritability. For low additivity ( $a^{2}$ value) and high heritability $\left(h_{H S}^{2}\right)$ HSP-RS is the best method.

The combination of the 2 kinds of fields in 3dimensional fields $\left[f\left(h_{H S}^{2} a^{2}, \rho_{L}\right)\right]$ obviously leads to the expected conclusion that $S_{1}-R S$ is the best method for medium to high value of $a^{2}$, for medium to high value of $\rho_{L}$ and from medium to low heritability $h_{H S}^{2}$.

Table II gives the relative efficiency (1 for HSP-RS) of the 4 methods for $h_{H S}^{2}$ varying from 0.05 to 0.50 with 3 values for $a^{2}(0.25,0.50$, $0.75)$ and 3 values for $\rho_{L}(0.50,0.70,0.90)$ corresponding to values for $\rho_{S 1}$ of $0.84,0.90,0.97$ ) with $r_{L}^{2} 1.50$. It shows that $S_{1}$-RS is the most efficient in a large range of situations and all the more so, as the heritability is lower $\left(S_{1}-R S\right.$ is $29 \%$ better than HSP-RS for $h_{H S}^{2} 0.30, a^{2}=$ 0.75 and $\rho_{L}=0.70$, and this superiority is $60 \%$ if $h_{H S}^{2}=0.10$ with the same values as previously for $a^{2}$ and $\left.\rho_{L}\right)$. When it is not the best, it is HSPRS that is the best (for low $a^{2}$ values, relatively low correlation $\rho_{L}$, and medium to high heritability). However, in the situations considered in table III, the inferiority of $S_{1}-R S$ is never strong (only $18 \%$ with $h_{H S}^{2}=0.50, a^{2}=0.25$ and $\rho_{L}=$ 0.50).

\section{Comparison on a per generation basis}

Figure 2 gives one example of the fields $f\left(h_{H S}^{2}\right.$, $\left.\rho_{L}\right)$ and $f\left(h_{H S}^{2}, a^{2}\right)$. The most striking fact is the quasi-general superiority of FSF-RS. Indeed, for the fields $f\left(h_{H S}^{2}, \rho_{L}\right)$ FSF-RS turns out to be the best for a high (and unrealistic) value of $h_{H S}^{2}$ only and for very low heritability and very high correlation $\rho_{L}$; for this situation $S_{1}$-RS is still the best. For the fields $f\left(h_{H S}^{2}, a^{2}\right)$ FSF-RS is not the best: only for low values of $a^{2}$ and medium to high heritability ; for that situation HSF-RS is the best method. In table II, the quasi-general advantage of FSF-RS clearly appears. In comparison to $S_{1}-R S$, its advantage varies approximately from 0 (for low $h_{H S}^{2}$ and high $\rho_{L}$ ) to $46 \%$ ( for 
Table II. Relative genetic advance of 4 methods of recurrent selection: 1) HSF-RS; 2) FSF-RS; 3 ) HSP-RS; 4) $\mathrm{S}_{1}-\mathrm{RS}$, for 3 levels of comparisons: A) per cycle; B) per generation; C) per unit of time (per year for an annual plant) with intercrossing in off-season; $r_{L}^{2}=1.50, p_{H S P}=0.20$. Such a computation has considered 80 plants per family and $S_{1}$ intrafamily correlation $(t)$ of 0.66 . This last parameter has little or no effect on the results. Forty plants per family also gives approximately the same results as 80 .

\begin{tabular}{|c|c|c|c|c|c|c|}
\hline $\mathrm{h}_{\mathrm{HS}}^{2}$ & $a^{2}$ & $\rho_{\mathrm{L}}$ & ${ }^{\rho} \mathrm{S} 1$ & $r_{L}^{2}$ & $\mathrm{~h}_{\mathrm{FS}}^{2}$ & $\mathrm{~h}_{\mathrm{S} 1}^{2}$ \\
\hline & & 30 & .67 & 0.68 & 0.08 & \\
\hline & & & & & & \\
\hline & & 90 & & 1.12 & 0.08 & \\
\hline & & & & & & \\
\hline & & & & & & \\
\hline & & & & 1.12 & 19 & \\
\hline & & & & & & \\
\hline & & & & 0.93 & 09 & \\
\hline & & & & & & \\
\hline & & & & 0.68 & 0.14 & \\
\hline & & & & & & \\
\hline & & & & & 0.14 & \\
\hline & & & & 0.68 & & \\
\hline & & 0.70 & 3 & 0.93 & 0.17 & 0. \\
\hline & & & & 1.12 & 17 & \\
\hline & & & & & & \\
\hline & & & & 0.93 & 0. & \\
\hline & & & & & & \\
\hline & & & & 0.68 & & \\
\hline & & & & & & \\
\hline & & 0.9 & & & & \\
\hline & & 0 & & 0 . & 9 & \\
\hline & & & & & & \\
\hline & & & & 1.1 & & \\
\hline & & & & 0.68 & & \\
\hline & & & & 0.93 & 0. & 0. \\
\hline & & & & 1.12 & 0 . & . \\
\hline & & & & 0. & 0 & \\
\hline & & & & & & \\
\hline & & & & 1. & & \\
\hline & & & & & & \\
\hline & & & & & 0 . & \\
\hline & & & & & & \\
\hline & & & & & 0 . & \\
\hline & & & & & & \\
\hline & & & & & & \\
\hline & & & & & & \\
\hline & & & & & 0.31 & \\
\hline & & & & & 0.3 & \\
\hline & & & & & & \\
\hline & & & & & & \\
\hline & & & & & & \\
\hline & & & & & 0.52 & \\
\hline & & & & & & \\
\hline & & & & & 0. & \\
\hline & & & & & & \\
\hline & & & & & & \\
\hline & & & & & 0. & \\
\hline & & & 0. & 0. & 0. & \\
\hline & & & & & 0. & \\
\hline & & & 0. & 1. & 0.5 & \\
\hline & & & & & & \\
\hline & & & 0.8 & 0. & 0.60 & \\
\hline & & & & & & \\
\hline
\end{tabular}

\begin{tabular}{llll}
\multicolumn{5}{c}{$(A)$} \\
\hline$(1)$ & $(2)$ & $(3)$ & $(4)$ \\
\hline 0.74 & 1.15 & 1.00 & 1.00 \\
0.74 & 1.15 & 1.00 & 1.42 \\
0.74 & 1.15 & 1.00 & 1.74 \\
0.74 & 1.20 & 1.00 & 1.04 \\
0.74 & 1.20 & 1.00 & 1.47 \\
0.74 & 1.20 & 1.00 & 1.80 \\
0.74 & 1.21 & 1.00 & 1.06 \\
0.74 & 1.21 & 1.00 & 1.49 \\
0.74 & 1.21 & 1.00 & 1.83 \\
0.74 & 1.06 & 1.00 & 0.92 \\
0.74 & 1.06 & 1.00 & 1.28 \\
0.74 & 1.06 & 1.00 & 1.56 \\
0.74 & 1.15 & 1.00 & 0.99 \\
0.74 & 1.15 & 1.00 & 1.37 \\
0.74 & 1.15 & 1.00 & 1.66 \\
0.74 & 1.18 & 1.00 & 1.01 \\
0.74 & 1.18 & 1.00 & 1.40 \\
0.74 & 1.18 & 1.00 & 1.70 \\
0.74 & 0.94 & 1.00 & 0.80 \\
0.74 & 0.94 & 1.00 & 1.10 \\
0.74 & 0.94 & 1.00 & 1.32 \\
0.74 & 1.06 & 1.00 & 0.90 \\
0.74 & 1.06 & 1.00 & 1.22 \\
0.74 & 1.06 & 1.00 & 1.45 \\
0.74 & 1.12 & 1.00 & 0.94 \\
0.74 & 1.12 & 1.00 & 1.26 \\
0.74 & 1.12 & 1.00 & 1.50 \\
0.74 & 0.85 & 1.00 & 0.72 \\
0.74 & 0.85 & 1.00 & 0.98 \\
0.74 & 0.85 & 1.00 & 1.17 \\
0.74 & 0.99 & 1.00 & 0.83 \\
0.74 & 0.99 & 1.00 & 1.11 \\
0.74 & 0.99 & 1.00 & 1.31 \\
0.74 & 1.06 & 1.00 & 0.88 \\
0.74 & 1.06 & 1.00 & 1.16 \\
0.74 & 1.06 & 1.00 & 1.36 \\
0.74 & 0.78 & 1.00 & 0.66 \\
0.74 & 0.78 & 1.00 & 0.89 \\
0.74 & 0.78 & 1.00 & 1.06 \\
0.74 & 0.94 & 1.00 & 0.77 \\
0.74 & 0.94 & 1.00 & 1.02 \\
0.74 & 0.94 & 1.00 & 1.20 \\
0.74 & 1.01 & 1.00 & 0.83 \\
0.74 & 1.01 & 1.00 & 1.08 \\
0.74 & 1.01 & 1.00 & 1.26 \\
0.74 & 0.73 & 1.00 & 0.61 \\
0.74 & 0.73 & 1.00 & 0.82 \\
0.74 & 0.73 & 1.00 & 0.98 \\
0.74 & 0.89 & 1.00 & 0.73 \\
0.74 & 0.89 & 1.00 & 0.95 \\
0.74 & 0.89 & 1.00 & 1.11 \\
0.74 & 0.97 & 1.00 & 0.78 \\
0.74 & 0.97 & 1.00 & 1.01 \\
0.74 & 0.97 & 1.00 & 1.17
\end{tabular}

(B)

(1) (2) (3) (4)

1.111 .721 .001 .00 1.111 .721 .001 .42 1.111 .721 .001 .74 1.110 .791 .001 .04 1.111 .791 .001 .47

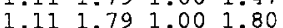
1.111 .821 .001 .06 1.111 .821 .0001 .06 1.111 .821 .0001 .49 1.111 .821 .001 .83 $\begin{array}{llll}1.11 & 1.59 & 1.00 & 0.92\end{array}$ 1.111 .591 .001 .28 1.111 .591 .001 .56 $\begin{array}{llll}1.11 & 1.72 & 1.00 & 0.99\end{array}$ $1.111 .72 \quad 1.001 .37$ $1.11 \quad 1.72 \quad 1.001 .66$ $1.111 .77 \quad 1.001 .01$ 1.111 .771 .001 .40 1.111 .771 .001 .70 1.111 .411 .000 .80 1.111 .411 .000 .80 1.31 .411 .001 .10 $1.11 \quad 1.411 .001 .32$ $1.11 \quad 1.591 .00 \quad 1.22$ 1.111 .591 .001 .45 $\begin{array}{llll}1.11 & 1.67 & 1.00 & 0.94 \\ 1.11 & 1.67 & 1.00 & 1.26\end{array}$ $1.11 \quad 1.67 \quad 1.001 .50$ $\begin{array}{llll}1.11 & 1.27 & 1.00 & 0.72\end{array}$ $\begin{array}{lllll}1.11 & 1.27 & 1.00 & 0.98\end{array}$ 1.111 .271 .001 .17 $1.11 \quad 1.491 .00 \quad 0.83$ $1.11 \quad 1.491 .00 \quad 1.11$ 1.121 .491 .001 .31 $1.111 .591 .00 \quad 0.88$ 1.111 .591 .001 .16 1.11 1.59 1.001 .36 1.113 .591 .001 .36 1.111 .171 .000 .69 1.111 .171 .000 .89 $\begin{array}{lllll}1.11 & 1.17 & 1.00 & 1.06\end{array}$ $\begin{array}{lllll}1.11 & 1.41 & 1.00 & 0.77\end{array}$ 1.111 .411 .001 .02 1.111 .411 .001 .20 1.111 .521 .000 .83 1.111 .521 .001 .08 1.111 .521 .001 .26 $\begin{array}{llll}1.11 & 1.09 & 1.00 & 0.61\end{array}$ $\begin{array}{llll}1.11 & 1.09 & 1.00 & 0.82\end{array}$

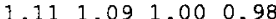
$1.11=331.000 .73$ 1.111 .331 .000 .73 1.111 .331 .000 .95 $1.111 .33 \quad 1.00 \quad 1.11$ 1.111 .461 .000 .78 $\begin{array}{llll}1.11 & 1.46 & 1.00 & 1.01 \\ 1.11 & 1.46 & 1.00 & 1.17\end{array}$
(C)

(1) (2) (3) (4)

1.472 .291 .001 .00 1.472 .291 .001 .42 $1.472 .291 .00 \quad 1.74$ 1.472 .391 .001 .04 1.472 .391 .0001 .04 1.472 .391 .001 .80 1.472 .431 .001 .06 1.477 .4331 .001 .049 1.477 .431 .001 .43 $\begin{array}{llll}1.47 & 2.12 & 1.000 & 0.92\end{array}$ 1.472 .121 .001 .28 1.472 .121 .001 .56 $1.472 .291 .00 \quad 0.99$ 1.472 .291 .001 .37 1.472 .291 .001 .66 $\begin{array}{llll}1.47 & 2.36 & 1.00 & 1.01\end{array}$ $\begin{array}{llll}1.47 & 2.36 & 1.00 & 1.40\end{array}$ 1.472 .361 .001 .70

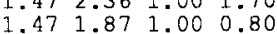
$1.471 .871 .00 \quad 1.80$ 1.471 .871 .001 .32 1.471 .871 .0001 .32 1.472 .121 .000 .90 $1.472 .121 .00 \quad 1.22$ 1.472 .121 .001 .45 $1.47 \quad 2.231 .000 .94$ $1.47 \quad 2.23 \quad 1.001 .26$ $\begin{array}{llll}1.47 & 2.23 & 1.00 & 1.50\end{array}$ $\begin{array}{llll}1.47 & 1.70 & 1.00 & 0.72\end{array}$ $1.471 .70 \quad 1.00 \quad 0.98$ $1.47 \quad 1.70 \quad 1.00 \quad 1.17$ $\begin{array}{llll}1.47 & 1.99 & 1.00 & 0.83\end{array}$ $1.471 .99 \quad 1.00 \quad 1.11$ $\begin{array}{llll}1.47 & 1.99 & 1.00 & 1.31\end{array}$ $1.472 .12 \quad 1.00 \quad 0.88$ $\begin{array}{llll}1.47 & 2.12 & 1.00 & 1.16\end{array}$ $1.472 .12 \quad 1.001 .36$ 1.471 .561 .000 .66 1.471 .561 .000 .89 1.471 .561 .001 .06 1.471 .871 .000 .77 1.471 .871 .001 .02 $1.47 \quad 1.87 \quad 1.00 \quad 1.20$ $1.472 .03 \quad 1.00 \quad 0.83$ 1.472 .031 .001 .08 $1.472 .03 \quad 1.001 .26$ $1.47 \quad 1.45 \quad 1.00 \quad 0.61$ $\begin{array}{lllll}1.47 & 1.45 & 1.00 & 0.82\end{array}$ $\begin{array}{llll}1.47 & 1.45 & 1.00 & 0.98\end{array}$ 1.471 .781 .000 .73 1.471 .781 .000 .95 1.471 .781 .001 .11 $\begin{array}{llll}1.47 & 1.78 & 1.00 & 1.11\end{array}$ 1.471 .951 .000 .78

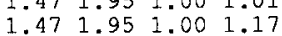

relatively high $h^{2}(0.50)$, high $a^{2}(0.75)$ and low $\left.\rho_{L}(0.50)\right)$.

Clearly the advantages of FSF-RS and HSF$\mathrm{RS}$ in some situations are due to their short cycle length and to their selection intensity being greater than for HSP-RS, due to the constraint of equal effective size. The consideration of the same selection intensity suppresses the possible advantage of HSF-RS (which appears to be the worst) but not that of FSF-RS.

\section{Comparisons on a per unit of time basis with one off-season generation for intercrossing}

In comparison with previous results (on a per generation basis) the use of one off-season generation for intercrossing suppresses the situations where $S_{1}-R S$ is the best, and increases the advantages of FSF-RS or HSF-RS. Indeed, their relative efficiencies are multiplied by 1.33 . 
Table III. Ratio of genetic advance by mass selection to that of full-sib family selection ( $r^{2}$ part of environmental variance due to variation among plots is 0.30 ).

\begin{tabular}{|c|c|c|c|c|}
\hline$h_{\mathrm{HS}}^{2} \quad \mathrm{a}^{2}$ & 0.20 & 0.50 & 0.70 & 1.00 \\
\hline 0.70 & 1.63 & 1.74 & 1.83 & 1.89 \\
\hline 0.50 & 1.45 & 1.55 & 1.63 & 1.68 \\
\hline 0.30 & 1.22 & 1.30 & 1.38 & 1.42 \\
\hline 0.15 & 1.02 & 1.07 & 1.12 & 1.15 \\
\hline 0.10 & 0.94 & 0.98 & 1.02 & 1.04 \\
\hline 0.05 & 0.85 & 0.88 & 0.90 & 0.91 \\
\hline
\end{tabular}

Table II shows that for $h_{H S}^{2}<0.50$ and $a^{2}>$ 0.25 , FSF-RS will always be the best method with a strong advantage on $S_{1}$-RS (from 20 to $90 \%$ ) and an advantage on HSF-RS from 0 to $56 \%$. The greater superiority on $\mathrm{S}_{1}-\mathrm{RS}$ is again for higher heritabilities.

The strong advantage of FSF-RS in comparison to HSP-RS or $\mathrm{S}_{1}$-RS is obviously due to the fact that for an annual plant the genetic advance per unit of time is multiplied by 2 , whereas that of HSP-RS and $S_{1}$-RS is multiplied by only 1.5 .

\section{Conclusions}

It is clear that according to the level of consideration of genetic advance, per cycle or per unit of time, with or without the use of one off-season generation for intercrossing, the best methods are not the same. On a per generation basis, without an off-season generation, the 2 competitive methods from medium to low heritabilities $\left(h_{H S}^{2}\right)$ are FSF-RS and $S_{1}$-RS. $S_{1}$-RS is a little more efficient than FSF-RS at low heritability (unrealistic low values of $h_{H S}^{2}$ ) and high correlation $\rho_{S_{1}}^{2}$. The superiority of FSF-RS for realistic values of $h_{H S}^{2}(0.30$ to 0.50 ) varies from $15 \%$ to $45 \%$ (for $\left.h_{H S}^{2}=0.50, a^{2}=0.75, \rho_{L}=0.50\right)$.

With the use of one off-season generation for intercrossing there is a clear advantage of FSFRS and the competitiveness of $S_{1}$-RS disappears. In such a situation, HSF-RS can be a competitive method but only for a relatively high $h_{H S}^{2}$ and low additivity $\left(a^{2} \leq 0.25\right)$.

In comparison to the result on a per cycle basis, the superiority of FSF-RS is mainly due to its short cycle length (only 2 generations with the possibility to develop pair-crossing in an offseason). The competitiveness of HSF-RS in some situations is mainly due to the high selec- tion intensity considered for this scheme in order to have the same effective size as with HSP-RS. However, with the same amount of means for testing the families, if 20 plants are selected for HSP-RS, only 5 families will be retained for HSF-RS, which is too low and will induce a great variation at the level of the realized genetic advance. It appears to be more realistic to have $p_{H S F}>p_{H S P} / 4$. An increase in rate of selection $p_{H S F}$ to values of $p_{H S P} / 2$ instead of $p_{H S P} / 4$ suppresses the competitiveness of HSF-RS.

Note that the parameters $\rho_{L}$ and $r_{L}^{2}$ affect only comparisons involving $S_{1}-R S$. Only results with $r_{L}^{2}=1.5$ have been related; lower values decrease the competitiveness of $S_{1}$-RS.

\section{COMPARISONS WITH MASS SELECTION}

As the methods using family or progeny testing have been previously compared, it is only necessary to compare mass selection with the best method on a per unit of time basis, that is to say to FSF-RS. Considering its practical interest in cross-fertilized plants, HSF-RS has also been chosen for the comparison. Even with the simplification on the number of plants per plot, the comparisons involving mass selection introduce 3 new parameters: the part $r^{2}$ of the variance between plots in the environmental variance, the number of replications $b$ and the number $N$ of plants per plot. Without assumption, it can be shown that FSF-RS will be better than mass selection, on a per unit of time basis, if the broad sense heritability is less than the following critical value :

$$
h_{c}^{2}=\frac{1 /\left(c^{2} j^{2}\right)-4 r^{2} / b-4\left(1-r^{2}\right) / N}{a^{2}(1-1 / N)+(1+3 / N)-4 r^{2} / b-4\left(1-r^{2}\right) / N}
$$

with $j=i_{M} / i_{F S}$ and $c=1 / 2$ with one off-season generation and 1 without off-season generation; $N$ is the number of plants per family.

Clearly when $N$ is sufficiently great, terms in $1 / N$ can be neglected. However, this does not reduce the number of parameters, because as the same number of plants is considered whatever the scheme of selection, $N$ affects the selection intensity $i_{M}$ because $p_{M}=p_{H S P} / N$.

Similarly, from the expressions of genetic advance given in table I, expressions of critical value of broad sense heritability have been established to have HSF-RS superior to mass selection, and HSF-RS superior to FSF-RS under the con- 

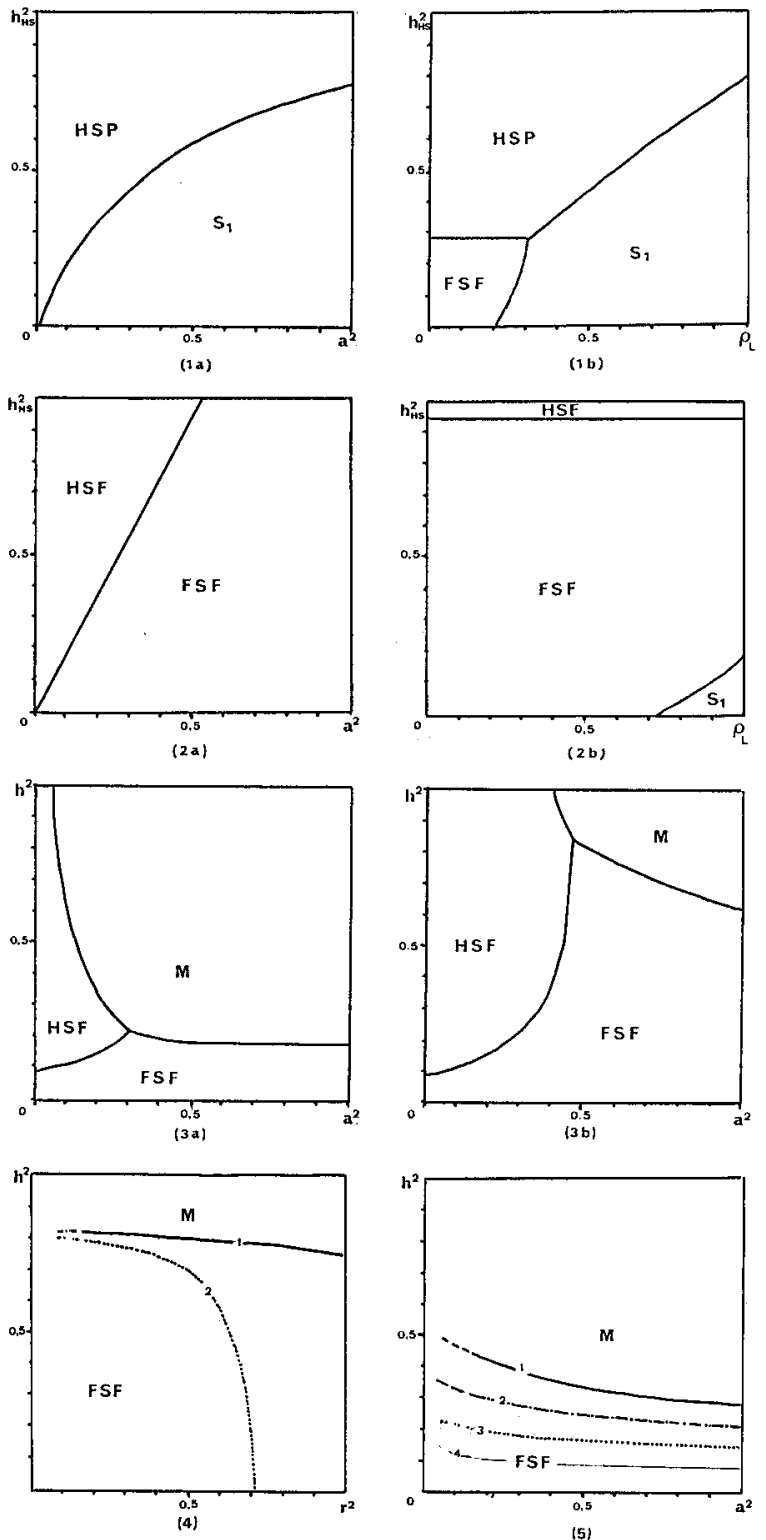

straints on the effective size. Relative genetic gains have also been computed for the same combinations of parameters. From the critical values of the broad sense heritability $\left(h^{2}\right)$, fields of efficiency $f\left(h^{2}, a^{2}\right)$ for given $a^{2}, b$ and $N$ and $f\left(h^{2}\right.$, $\left.a^{2}\right)$ for given $r^{2}, b$ and $N$ have been established. Some examples are given in figure 3 . It has been verified that the simplification due to a sufficiently large number of plants per plots is acceptable.

\section{Fields of efficiency $f\left(\mathrm{~h}^{2}, \mathrm{a}^{2}\right)$}

\section{Without an off-season generation}

Without an off-season generation, with $b$ varying between 2 to 8 , mass selection is always better
Figs 1-5. Fields of efficiency of the breeding methods studied (HS or FSF or $\mathrm{P}=$ half-sib or full-sib family on progeny recurrent selection, $S_{1}=S_{1}$ progeny (or family) recurrent selection, $M=$ mass selection). $h_{H S}^{2}=$ heritability at the level of HS family; $a^{2}=$ part of additive variance in the total genetic variance; $\rho_{\mathrm{L}}$ correlation between additive effects for per se value and line value; $r^{2}=$ part of environmental variance due to variation between plots. When a method is the most efficient, its name is written in the domain delimited by thick traits or curves ; broken boundaries correspond either to unrealistic values of parameters or to values for which the approximation on heritabilities induces an error but does not qualitatively change the results. Figs 1 and 2 . Fields of efficiency for methods with progeny or family testing. In the comparisons involving $S_{1}-R S$, the ratio between additive variance in line value to additive variance for the per se value $\left(r_{t}^{2}\right)$ is 1.5. 1. For genetic advance per cycle. a) with $=0.70 ; b$ ) with $a^{2}=0.50$. 2. For genetic advance per generation. a) with $r_{t}^{2}=$ $0.70, b)$ with $a^{2}=0.50$. Figs 3 and 4 . Comparisons involving mass selection. $h^{2}$ is the broad sense heritability in a site of selection and $b n=80$. Fig 3a. Without off-season generation for family selection and $r^{2}=0.30$. 1) with complete randomization; (2) $b=8$. Fig 3b. For $r^{2}=0.30$, with one off-season generation. (1) and (2) as in 3a. Fig 4. For $a^{2}=0.50$, with one off-season generation. (1) $b=8$ and (2) $b=2$. Fig 5 . Comparison between mass selection and FSF-RS in presence of $G \times E$ interaction (with $r^{2}$ part of environmental variation due to plot in a site equal to $0.30, p_{H S}=0.20$, number of plants per family in a site of FSF $b n=80$, part of $G \times E$ interaction variance in the total genetic variance of 0.75 due to dominance for $75 \%)$. (1) with $b=l$ and $l=8$, (2) with $b=2$ and $I=4$, (3) with $b=4$ and $I=2$ ( $l$ being the number of testing sites). Note that the consideration of a constant total number of plants ( 160 or 320 ) per family in FSF-RS does not greatly change the representation.

than HSF-RS (fig 3a). It is also better than FSF$R S$ in a large range of realistic situations. FSF$\mathrm{RS}$ is better than mass selection only at very low broad sense heritability $\left(h^{2}<0.10\right)$ for low values of $a^{2}$, a high number of replications $(b=8)$, and relatively low $r^{2}$ value $\left(r^{2}=0.30\right)$. Even in these situations the inferiority of FSF-RS is only $\approx 10 \%$, a value which contrasts with the superiority of 30 $-40 \%$ of mass selection for medium heritability $\left(h^{2}=0.30-0.40\right)$ and average value of $a^{2}$ (table III).

For the same number of replications, the change in the number of plants per family does not affect the results very much. Forty plants give approximately the same domains of efficiency as 80 plants: the number of replications is more important that the number of plants. However, if 
the number of plants per family decreases this tends to enlarge the field of efficiency of FSF-RS because, under the constraints, this decreases the selection intensity for mass selection. With complete randomization of the plants (such a design is used in tree improvement) the field of efficiency of FSF-RS is slightly enlarged (fig 3a).

The relatively large and general advantage of mass selection is obviously due to the high selection intensity considered as a consequence of constraints on the number of plants studied and on the effective size. With $p_{H S P}=0.20, p_{F S F}=$ 0.10 and with 80 plants per family $p_{M}=0.0025$, then $i_{F S F}=1.755$ and $i_{M}=3.10$. If $p_{M}$ is taken to be $>p_{H S} / N$ but significantly $<p_{H S}$ (for example, $p_{M}=2 p_{H S}(M)$, the field of efficiency of mass selection is slightly narrowed.

\section{With one off-season generation}

With one off-season generation, figure $3 b$ shows the field $f\left(h^{2}, a^{2}\right)$ for $r^{2}=0.30, b=8$ and $N=80$. The superiority of FSF-RS appears for a large range of realistic situations. For a heritability of $0.30-0.40 \%$, the advantage of FSF-RS over mass selection is $\approx 30 \%$ (table II). Mass selection is the best only at relatively high broad sense heritability and high values of $a^{2}$ (which corresponds to the classically admitted domain of efficiency of mass selection). HSF-RS is the best for a high broad sense heritability but a low value of $r^{2}$ (ie for situations of relatively low narrow sense heritability due to a high dominance variance). A decrease in the number of replications enlarges the field of efficiency of mass selection and narrows that of HSF-RS.

The increase in the number of plants per family $(N)$ and the decrease in the number of replications narrows the field of efficiency of FSF-RS because with the given constraints the ratio $i_{M} / i_{F S}$ increases when $N$ increases and $h_{F S}^{2}$ decreases when $b$ decreases. However, the shape of the fields remains approximately the same.

\section{Fields of efficiency $f\left(\mathrm{~h}^{2}, \mathrm{r}^{2}\right)$}

\section{Without an off-season generation}

Without an off-season generation, it again appears that mass selection is the best method for a large range of realistic situations. FSF-RS is better than mass selection only for very low broad sense heritability and low $r^{2}$ values. This domain of efficiency increases when the number of replications increases, because it tends to suppress the effect of $r^{2}$. FSF-RS is always better than HSF-RS.

\section{With one off-season generation}

With one off-season generation, figure 4 shows the fields of efficiency for $a^{2}=0.50$. It appears that with a relatively high number of replications $(b=8)$ mass selection is the best method only at high broad sense heritability $\left(h^{2}>0.80\right.$ for $b=$ 8). However, with a low number of replications and high value of $r^{2}$, mass selection is the best. This is due to the fact that for a high correlation due to environment among neighbouring plants it is preferable to randomize plants of the same family.

\section{Discussion}

Factors affecting the comparisons of mass selection to other schemes are the following:

Selection intensity. With the given constraints and assumptions, mass selection is very often the best method. This is due to the high possible selection intensity imposed by the conditions: 1) the same number of plants for a nursery with mass selection as with the trials with progeny or family testing ; 2) the same number of plants selected. Obviously if the number of plants observed for mass selection is decreased, or if a greater number of plants are selected, the field of efficiency of FSF-RS will enlarge.

The selection of parents of the progenies. Without an off-season generation it could be also argued that with family or progeny testing it is possible to select the progenitors for some characters before crossing. Then if such characters are correlated to characters evaluated in trials, the genetic advance is FSF-RS or HSP-RS could be increased. This will enlarge the field of efficiency of FSF-RS but does not appear sufficient to qualitatively change the results. With an off-season generation it will be difficult or even impossible to correctly select the parents; it could be better to have no selection, and then the developed comparisons would be valid.

The reduction in variance due to selection. Another fact tends to enlarge the field of efficiency of FSF-RS by decreasing the efficiency of mass selection: the decrease in genetic variance after 1 and 2 cycles of selection. However, assuming 
only additive effects using Bulmer's formula $\Delta \sigma_{A}^{2} /$ $\sigma_{A}^{2}=i_{M}\left(i_{M}-x_{M}\right) h^{2} / 2$ to predict the decrease leads to a decrease in genetic variance of only $\approx 5 \%$ for $h^{2}=0.10$ and $p_{M}=2 \%$ o $\left(i_{M}=3.17 ; x_{M}=\right.$ 2.88); for $h^{2}=0.30$, the decrease will be $15 \%$. Obviously the decrease is all the greater the higher the heritability. Then, without an offseason generation, for high heritability this will significantly reduce the expected degree of superiority of mass selection in comparison to FSFRS, but will not significantly change the results for low heritability. Note, however, that in the previous example, with $h^{2}=0.30$, the reduction in genetic advance by mass selection will be only $5 \%$; it will be in the order of $14 \%$ for $h^{2}=0.60$. Considering the degree of superiority of mass selection for medium to high heritability, this will not greatly modify its field of efficiency. With one off-season generation for intercrossing, the length of the cycles is the same for mass selection as for FSF-RS, then on the basis of the comparison of the first cycle, it is not necessary to consider the difference in the reduction of variance.

The existence of a $G \times E$ interaction. A further factor that can decrease the efficiency of mass selection is the presence of genotype $x$ environment ( $G \times E)$ interactions. It is clear that if mass selection is developed in only one site it does not consider $G \times E$ interaction as well as with multisite FSF-RS or HSP-RS. However, even in this situation, due to its shorter cycle mass selection will independently sample 2 or 3 environments, and this can counterbalance its expected inferiority. Furthermore, if mass selection in one site is compared with FSF-RS with several sites for testing, in order to have a comparison with the same number of plants tested, the selection intensity for mass selection can be increased, and $p_{M}=p_{H S P} /(I M)$, where $l$ is the number of sites. For example, with a selection rate of $p_{H S P}$ of $20 \%\left(i_{H P}=1.40\right)$ for HSP-RS and 40 plants per progeny in a site, the rate of selection for mass selection will be $0.5 \%\left(i_{M}=2.89\right)$. With 3 sites for testing with HSP-RS, the rate of mass selection can be decreased to $0.16 \%$ and then the selection intensity will be increased to $3.23(+12 \%)$. This could again, at least partially, counterbalance the expected inferiority of mass selection due to selection in only one site. Note that the increase in efficiency of mass selection by increasing the selection intensity is all the weaker as the primary selection intensity is the higher.

Another way to increase the efficiency of mass selection in the presence of $G \times E$ interac- tion is to develop convergent-divergent selection as proposed by Lonnquist (1979). In this case, for the same population, independent selections are developed in each site and the selected units are mixed to produce the next generation which is again distributed in each site. Clearly, this allows one to take into consideration $\mathrm{G} \times \mathrm{E}$ interactions in mass selection and can give to it an advantage due to its short cycle. Without more theoretical development it is difficult to predict whether the field of efficiency of mass selection will be enlarged or narrowed.

Note that for a plant like maize, it can be difficult to take into consideration $G \times E$ interaction with FSF-RS, because one ear will not give enough seeds. In this case convergent-divergent FSF-RS can also be developed.

The structure of the experimental design. In the comparison of mass selection to other schemes with family and progeny testing an important parameter appears: the environmental correlation $\left(r^{2}\right)$ among plants of the same plot. This is also the percent of the total environmental variance due to the plots. If $r$ is low, this enlarges the field of efficiency of the methods with progeny or family testing; this is the same effect as an increase in the number of replications. For the opposite high $r^{2}$ values the field of efficiency of mass selection is enlarged, mainly for a low number of replications (fig 4). To suppress or at least decrease the unfavourable effect of $r^{2}$ with family or progeny testing, for a given number of plants per family, it will be necessary to increase the number of replications and then the optimum will be one plant per plot. While this is possible for forest or fruit trees, it seems to be difficult to conceive such an experimental design for maize, or beets, not only by consideration of the effect of competition, but also mainly from a practical point of view.

Note that if the breeder accepts such an experimental design, then it is necessary to again reconsider the use of mass selection. Indeed if the plants are identified according their parental origin, it is possible to use a combined individualfamily selection. This can greatly increase the efficiency of mass selection for low heritabilities. This is also possible with a plot structure but will not be so efficient.

The use of associated characters. It should be also mentioned that the use of associated characters to increase the efficiency of the selection will be more efficient for mass selection than for those schemes with family or progeny testing be- 
cause associated characters are the more efficient the lower the heritability. Molecular markers can also be used with the same objective (Lande and Thompson, 1990) and are also the more efficient the lower the heritability for the primary character.

\section{CONCLUSION}

The theoretical approach developed here allows the determination of the fields of efficiency of various recurrent selection methods aimed at improving the per se value or the combining ability of a population. To simplify the determination for methods with family or progeny testing, it has been assumed that the total number of plants per family was sufficiently great ( $>50$ ). This will generally be satisfied when the families are evaluated in plots with $>10$ plants per plot with 3 to 5 replications. Obviously this leads to an approximation for the determination of the fields of efficiency: their boundaries are not known with accuracy. However, with $N>50$ the error in the determination will be low in realistic situations and can be neglected, because for the breeder to adopt another method that can be more expensive or more difficult to use, the relative efficiency must be sufficiently great (>20\%). Furthermore the whole study assumes the absence of competition, ie, the independence between the plants within a plot. This is a classical assumption in quantitative genetics. It is possible to remove such an assumption (Griffing 1967, Gallais 1975, 1976) ; however, the number of parameters then increases greatly. A more empirical approach consists of assuming that the theory of covariances between relatives remains valid under such conditions. For methods with family or progeny testing, this appears quite possible when the performances of a set of families are about the same if tested in a mixture or in a monoculture. This has been experimentally verified by Rotili et al (1976) in alfalfa and by Gallais (1975) in cocksfoot. The absence of $\mathrm{G} \times \mathrm{E}$ interactions has also been assumed in this study, not only for simplification, but also to have a reference to study the effect of $G \times E$ interactions via the same approach. It is quite possible to study the effect of $\mathrm{G} \times \mathrm{E}$ interactions at the level of all the methods studied except $S_{1}$-RS by the introduction of 3 news parameters: the part of the phenotypic variance due to $G \times E$ interaction, the contribution of additive genetic effects to this part of the variation and the number of sites. It will be expected that the comparisons more affected by $\mathrm{G} \times \mathrm{E}$ interactions will be those involving mass selection (Gallais, unpublished results). Figure 5 gives one example of the comparison of mass selection to FSF-RS in the presence of relatively strong $G \times E$ interaction without the use of one off-season generation: as expected, the field of efficiency of FSF-RS is enlarged; however, due to the high selection intensity considered for mass selection, this scheme remains the most efficient over a large range of situations. Furthermore, the theoretical results of Dhillon (1991) on the comparison of the classical mass selection of $S_{0}$ plants to mass selection of $S_{1}$ also plants support the conclusion that $G \times E$ interactions have a relatively low effect on the relative efficiency of breeding methods of the same type (with progeny or family testing in our study).

Such assumptions allow a relatively simple determination of the fields of efficiency for methods involving family or progeny testing. The main parameters that must be known are the heritability (broad sense, at the individual level, or for half-sib families), the part of additive variance in the total genetic variance, and the part of the environmental variance due to the variation among plots. With the assumption of a sufficiently great number of plants per plot, this last parameter will be the main component of the residual of a trial with progenies. To compare with the $S_{1}-R S$ it is necessary to know 2 more parameters: $r_{s_{1}}^{2}$, the ratio of additive variance for $S_{1}$ value to the classical additive variance and $\rho_{S_{1}}$ the correlation between additive effect per se value and additive effect for $S_{1}$ value.

In comparison to results already known, the approach developed here allows a more accurate determination of the fields of efficiency of each method. It shows how the ranking of the breeding methods can be affected by the level of expression of genetic advance: per cycle and per unit of time, with or without an off-season generation for intercrossing. Considering only methods with family and progeny testing on a per cycle basis, methods with progeny testing are favored: FSF-RS is the best method or nearly the best except in situations with a high $\rho_{S 1}$ in which case $S_{1}-R S$ is the best. However, per unit of time, full-sib family selection tends to be one of the best methods, with or without an offseason generation. With one off-season generation the superiority of FSF-RS over the second can be $>50 \%$. As far as comparisons with mass selection are involved, it appears that mass selection can be competitive with family or progeny 
selection without an off-season generation, for medium to low heritabilities but not with one offseason generation. Furthermore, as it is possible to increase the efficiency of phenotypic selection in several ways (combined selection, convergent-divergent selection, associated characters, markers, etc) it appears that mass selection or its improved versions can be very competitive with progeny or family selection except in the case of extremely low heritability.

\section{REFERENCES}

Bulmer MG (1980) The Mathematical Theory of Quantitative Genetics. Academic Press, Washington, 4th edn

Choo PM, Kannenberg LW (1979) Relative efficiencies of population improvement in corn: a simulation study. Crop Sci 19, 179-185

Dhillon BS (1991) Recurrent mass selection based on selfed evaluation in allogamous species. Crop Sci 31, 1075-1077

Gallais A (1975) Sur quelques aspects de la compétition en amélioration des plantes. Ann Amélior Plantes 25, 51-64

Gallais A (1976) Effects of competition on means, variances and covariances in quantitative genetics with an application to general combining ability selection. Theor Appl Genet 47, 189-195

Gallais A (1988) Prediction of the mean and variance of lines and hybrids that can be derived from a random mating population. Theor Appl Genet 75, 625627

Gallais A (1989) Théorie de la Sélection en Amélioration des Plantes. Masson, Paris, pp 588

Gallais A (1990) Application of the concepts of test value and varietal value to the study of genetic ad- vance in recurrent selection: a synthesis. Euphytica 48, 197-209

Griffing B (1967) Selection in reference biological groups. I. Individual and group selection applied to population of unordered groups. Aust J Biol Sci 10, 127-139

Gillois M (1964) La relation d'identité en génétique. Thèse Fac Sci Paris

Hallauer AR, Miranda JB (1981) Quantitative Genetics in Maize Breeding. lowa State Univ Press, IA, pp 468

Harris DL (1964) Genotypic covariances between inbred relatives. Genetics 50, 1319-1348

Jones LP, Compton WA, Gardner CO (1971) Comparison of full and half-sib reciprocal selection. Theor App/ Genet 41, 36-39

Lande R, Thompson R (1990) Efficiency of marker assisted selection in the improvement of quantitative trait. Genetics 124, 743-756

Lonnquist JH, Compton WA, Gaedelmann JL, Loeffel FA, Shank Boyd, Troyer AF (1979) Convergentdivergent selection for area improvement in maize. Crop Sci 19, 602-604

Rotili P, Zannone L, Jacquard P (1976) Effects of association on the evaluation of lucerne populations. Ann Amélior Plantes 26, 139-155

Sprague GF (196) Quantitative genetics in plant improvement. In: Plant Breeding (KJ Frey, ed) University Press, Ames, IA, 315-354

Verrier E, Colleau JJ, Foulley JL (1990) Predicting cumulated response to directional selection in finite panmictic populations. Theor Appl Genet 79, 833840

Wright AJ (1981) A comparison of the expected efficiencies of some methods of population improvement. In: Quantitative Genetics and Breeding Methods: Proc 4th Meeting of the Section Eucarpia Biometrics in Plant Breeding (Gallais A, ed) INRA Versailles, 31-40 